\title{
Shearlet approximations to the inverse of a family of linear operators
}

Lin $\mathrm{Hu}^{1,2}$ and Youming $\mathrm{Liu}^{1 *}$

"Correspondence:

liuym@bjut.edu.cn

'Department of Applied

Mathematics, Beijing University of

Technology, Beijing, 100124, P.R.

China

\begin{abstract}
The Radon transform plays an important role in applied mathematics. It is a fundamental problem to reconstruct images from noisy observations of Radon data. Compared with traditional methods, Colona, Easley and etc. apply shearlets to deal with the inverse problem of the Radon transform and receive more effective reconstruction. This paper extends their work to a class of linear operators, which contains Radon, Bessel and Riesz fractional integration transforms as special examples. MSC: $42 \mathrm{C} 15 ; 42 \mathrm{C} 40$
\end{abstract}

Keywords: inverse problems; shearlets; approximation; Radon transform; noise

\section{Introduction and preliminary}

The Radon transform is an important tool in medical imaging. Although $f \in L^{1}\left(\mathbb{R}^{2}\right)$ can be recovered analytically from the Radon data $R f(\theta, t)$, the solution is unstable and those data are corrupted by some noise in practice [1]. In order to recover the object $f$ stably and control the amplification of noise in the reconstruction, many methods of regularization were introduced including the Fourier method, singular value decomposition, etc. [2]. However, those methods produced a blurred version of the original one.

Curvelets and shearlets were then proposed, which proved to be efficient in dealing with edges [3-7]. In 2002, Candés and Donoho applied curvelets [5] to the inverse problem

$$
Y=R f+\varepsilon W
$$

where the recovered function $f$ is compactly supported and twice continuously differentiable away from a smooth edge; $W$ denotes a Wiener sheet; $\varepsilon$ is a noisy level. Because curvelets have complicated structure, Colonna, Easley, etc. used shearlets to deal with the problem (1.1) in 2010 and received an effective reconstructive algorithm [8].

Note that the Bessel transform and the Riesz fractional integration transform arise in many scientific areas ranging from physical chemistry to extragalactic astronomy. Then this paper considers a more general problem,

$$
Y=K f+\varepsilon W,
$$

\section{Springer}

(c) $2013 \mathrm{Hu}$ and Liu; licensee Springer. This is an Open Access article distributed under the terms of the Creative Commons Attribution License (http://creativecommons.org/licenses/by/2.0), which permits unrestricted use, distribution, and reproduction in any medium, provided the original work is properly cited. 
where $K$ stands for a linear operator mapping the Hilbert space $L^{2}\left(\mathbb{R}^{2}\right)$ to another Hilbert space $Y$ and satisfies

$$
\left(K^{\prime \prime} K f\right)^{\wedge}(\xi)=\left(b+|\xi|^{2}\right)^{-\alpha} \hat{f}(\xi)
$$

with $b>0, \alpha>0$ ( $K^{\prime \prime}$ is the conjugate operator of $\left.K\right)$. Here and in what follows, $\hat{f}$ denotes the Fourier transform of $f$. The next section shows that Radon, Bessel and Riesz fractional integration transforms satisfy the condition (1.3).

The current paper is organized as follows. Section 2 presents three examples for (1.3) and several lemmas. An approximation result is proved in the last section, which contains Theorem 4.2 of [8] as a special case.

At the end of this section, we introduce some basic knowledge of shearlets, which will be used in our discussions. The Fourier transform of a function $f \in L^{1}\left(\mathbb{R}^{2}\right) \cap L^{2}\left(\mathbb{R}^{2}\right)$ is defined by

$$
\hat{f}(\xi)=\int_{\mathbb{R}^{2}} f(x) e^{-2 \pi i x \cdot \xi} d x
$$

The classical method extends that definition to $L^{2}\left(\mathbb{R}^{2}\right)$ functions.

There exist many different constructions for discrete shearlets. We introduce the construction [8] by taking two functions $\psi_{1}, \psi_{2}$ of one variable such that $\hat{\psi}_{1}, \hat{\psi}_{2} \in C^{\infty}(\mathbb{R})$ with their supports supp $\hat{\psi}_{1} \subset\left[-\frac{1}{2},-\frac{1}{16}\right] \cup\left[\frac{1}{16}, \frac{1}{2}\right]$, supp $\hat{\psi}_{2} \subset[-1,1]$ and

$$
\sum_{j \geq 0}\left|\hat{\psi}_{1}\left(2^{-2 j} \omega\right)\right|^{2}=1 \quad\left(|\omega| \geq \frac{1}{8}\right), \quad \sum_{l=-2^{j}}^{2^{j}-1}\left|\hat{\psi}_{2}\left(2^{j} \omega-l\right)\right|^{2}=1 \quad(|\omega| \leq 1) .
$$

Here, $C^{\infty}\left(\mathbb{R}^{n}\right)$ stands for infinitely many times differentiable functions on the Euclidean space $\mathbb{R}^{n}$. Then two shearlet functions $\psi^{(0)}, \psi^{(1)}$ are defined by

$$
\hat{\psi}^{(0)}(\xi):=\hat{\psi}_{1}\left(\xi_{1}\right) \hat{\psi}_{2}\left(\frac{\xi_{2}}{\xi_{1}}\right) \quad \text { and } \quad \hat{\psi}^{(1)}(\xi):=\hat{\psi}_{1}\left(\xi_{2}\right) \hat{\psi}_{2}\left(\frac{\xi_{1}}{\xi_{2}}\right)
$$

respectively.

To introduce discrete shearlets, we need two shear matrices

$$
B_{0}=\left(\begin{array}{ll}
1 & 1 \\
0 & 1
\end{array}\right), \quad B_{1}=\left(\begin{array}{ll}
1 & 0 \\
1 & 1
\end{array}\right)
$$

and two dilation matrices

$$
A_{0}=\left(\begin{array}{ll}
4 & 0 \\
0 & 2
\end{array}\right), \quad A_{1}=\left(\begin{array}{ll}
2 & 0 \\
0 & 4
\end{array}\right) .
$$

Define discrete shearlets $\psi_{j, l, k}^{(d)}(x):=2^{\frac{3}{2} j} \psi^{(d)}\left(B_{d}^{l} A_{d}^{j} x-k\right)$ for $j \geq 0,-2^{j} \leq l \leq 2^{j}-1, k \in \mathbb{Z}^{2}$ and $d=0,1$. Then there exists $\hat{\varphi} \in C_{0}^{\infty}\left(\mathbb{R}^{2}\right)$ such that

$$
\left\{\varphi_{j_{0}, k}(x), \psi_{j, l, k}^{(d)}(x), j \geq j_{0} \geq 0,-2^{j} \leq l \leq 2^{j}-1, k \in \mathbb{Z}^{2}, d=0,1\right\}
$$


forms a Parseval frame of $L^{2}\left(\mathbb{R}^{2}\right)$, where $\varphi_{j_{0}, k}(x):=2^{j_{0}} \varphi\left(2^{j_{0}} x-k\right)$. More precisely, for $f \in$ $L^{2}\left(\mathbb{R}^{2}\right)$,

$$
f(x)=\sum_{k \in \mathbb{Z}^{2}}\left\langle f, \varphi_{j_{0}, k}\right\rangle \varphi_{j_{0}, k}(x)+\sum_{d=0}^{1} \sum_{j \geq j_{0}} \sum_{l=-2^{j}}^{2^{j}-1} \sum_{k \in \mathbb{Z}^{2}}\left\langle f, \psi_{j, l, k}^{(d)}\right\rangle \psi_{j, l, k}^{(d)}(x)
$$

holds in $L^{2}\left(\mathbb{R}^{2}\right)$. It should be pointed out that $\psi_{j, l, k}^{(d)}(x)$ are modified for $l=-2^{j}$ and $2^{j}-1$, as seen in [8].

\section{Examples and lemmas}

In this section, we provide three important examples of a linear operator $K$ satisfying $\left(K^{*} K f\right)^{\wedge}(\xi)=\left(b+|\xi|^{2}\right)^{-\alpha} \hat{f}(\xi)$ and present some lemmas which will be used in the next section. To introduce the first one, define a subspace of $L^{2}\left(\mathbb{R}^{2}\right)$,

$$
D\left(\mathbb{R}^{2}\right):=\left\{f \in L^{1}\left(\mathbb{R}^{2}\right), f \text { is bounded }\right\} \subseteq\left\{f \in L^{2}\left(\mathbb{R}^{2}\right), \int_{\mathbb{R}^{2}}|\xi|^{-1}|\hat{f}(\xi)|^{2} d \xi<+\infty\right\}
$$

and a Hilbert space

$$
L^{2}([0, \pi) \times \mathbb{R}):=\left\{f(\theta, t), \int_{0}^{\pi} \int_{\mathbb{R}}|f(\theta, t)|^{2} d t d \theta<+\infty\right\}
$$

with the inner product $\langle f, g\rangle:=\int_{0}^{\pi} \int_{\mathbb{R}} f(\theta, t) \overline{g(\theta, t)} d t d \theta$.

Example 2.1 Let $L_{\theta, t}:=\{(x, y), x \cos \theta+y \sin \theta=t\} \subseteq \mathbb{R}^{2}$ and $d s(x, y)$ be the Euclidean measure on the line $L_{\theta, t}$. Then the classical Radon transform $\mathbb{R}: D(\mathbb{R}) \rightarrow L^{2}([0, \pi) \times \mathbb{R})$ defined by

$$
R f(\theta, t)=\int_{L_{\theta, t}} f(x, y) d s(x, y)
$$

satisfies $\left(R^{*} R f\right)^{\wedge}(\xi)=|\xi|^{-1} \hat{f}(\xi)$.

Proof By the definition of $D\left(\mathbb{R}^{2}\right), \int_{\mathbb{R}^{2}}|\xi|^{-1} \hat{f}(\xi) \overline{\hat{g}(\xi)} d \xi<+\infty$ for $f, g \in D\left(\mathbb{R}^{2}\right)$. It is easy to see that $\int_{0}^{2 \pi} d \theta \int_{0}^{+\infty} \hat{f}(\omega \cos \theta, \omega \sin \theta) \overline{\hat{g}(\omega \cos \theta, \omega \sin \theta)} d \omega=\int_{0}^{\pi} d \theta \int_{\mathbb{R}} \hat{f}(\omega \cos \theta, \omega \sin \theta) \times$ $\overline{\hat{g}(\omega \cos \theta, \omega \sin \theta)} d \omega$. This with the Fourier slice theorem ([1, 9]) and the Plancherel formula leads to

$$
\begin{aligned}
\int_{\mathbb{R}^{2}}|\xi|^{-1} \hat{f}(\xi) \overline{\hat{g}(\xi)} d \xi & =\int_{0}^{2 \pi} d \theta \int_{0}^{+\infty} \hat{f}(\omega \cos \theta, \omega \sin \theta) \overline{\hat{g}(\omega \cos \theta, \omega \sin \theta)} d \omega \\
& =\int_{0}^{\pi} d \theta \int_{\mathbb{R}}\left(R_{\theta} f\right)^{\wedge}(\omega) \overline{\left(R_{\theta} g\right)^{\wedge}(\omega)} d \omega \\
& =\int_{0}^{\pi} d \theta \int_{\mathbb{R}} R f(\theta, t) \overline{R g(\theta, t)} d t=\langle R f, R g\rangle,
\end{aligned}
$$

where $R_{\theta} f(t):=R f(\theta, t)$. Moreover, $\left\langle\left(R^{*} R f\right)^{\wedge}, \hat{g}\right\rangle=\left\langle R^{*} R f, g\right\rangle=\langle R f, R g\rangle=\left\langle|\xi|^{-1} \hat{f}(\xi), \hat{g}(\xi)\right\rangle$ for each $g \in D\left(\mathbb{R}^{2}\right)$. 
Because $D\left(\mathbb{R}^{2}\right)$ is dense in $L^{2}\left(\mathbb{R}^{2}\right)$, one receives the desired conclusion $\left(R^{\prime \prime} R f\right)^{\wedge}(\xi)=$ $|\xi|^{-1} \hat{f}(\xi)$. Here, $R^{*} R f \in L^{2}\left(\mathbb{R}^{2}\right)$ for $f \in D\left(\mathbb{R}^{2}\right)$. In fact, $R^{\prime \prime} R f=4 \pi I_{1} f$ by [10], where $I_{1}$ is the Riesz fractional integration transform defined by

$$
I_{1}(f)(x):=C_{\alpha} \int_{\mathbb{R}^{2}} \frac{f(x-y)}{|y|} d y
$$

with some normalizing constant $C_{\alpha}$ [11]. We rewrite $I_{1}(f)(x)=\int_{|y| \leq r} \frac{f(x-y)}{|y|} d y+$ $\int_{|y|>r} \frac{f(x-y)}{|y|} d y=: J_{1}+J_{2}$. Let $h(y)=\frac{1}{|y|} 1_{B(0,1)}(y), h_{r}(y)=\frac{1}{r^{2}} h\left(\frac{y}{r}\right)$, where $B(0,1)$ stands for the unit ball of $\mathbb{R}^{2}$ and $1_{A}$ represents an indicator function on the set $A$. Then $J_{1}=\int_{|y| \leq r} \frac{f(x-y)}{|y|} d y=$ $r \int_{|y| \leq r} h_{r}(y) f(x-y) d y \leq r M f(x)$ by Theorem 9 of reference [12, p.59], where $M f$ is the Hardy-Littlewood maximal function of $f$.

On the other hand, the Holder inequality implies

$$
J_{2} \leq\|f\|_{\frac{p}{p-1}}\left(\int_{|y|>r} \frac{1}{|y|^{p}} d y\right)^{\frac{1}{p}} \leq r^{2-p}\|f\|_{\frac{p}{p-1}}
$$

with $p>3$. Take $r=[M f(x)]^{-\frac{1}{p-1}}$, one gets $I_{1}(f)(x) \leq[M f(x)]^{\frac{p-2}{p-1}}\left(1+\|f\|_{\frac{p}{p-1}}\right)$ and $\left\|I_{1}(f)\right\|_{2} \leq$ $\left(1+\|f\|_{\frac{p}{p-1}}\right)\|M f\|_{\frac{2(p-2)}{p-1}}^{\frac{p-2}{p-1}} \lesssim\left(1+\|f\|_{\frac{p}{p-1}}\right)\|f\|_{\frac{2(p-2)}{p-1}}^{\frac{p-2}{p-1}}<+\infty$, since $f \in L^{\frac{p}{p-1}}\left(\mathbb{R}^{2}\right) \cap L^{\frac{2(p-2)}{p-1}}\left(\mathbb{R}^{2}\right)$ due to the assumption $f \in D\left(\mathbb{R}^{2}\right)$ and $\frac{2(p-2)}{p-1}>1, \frac{p}{p-1}>1$.

In order to introduce the next example, we use $f * g$ to denote the convolution of $f$ and $g$.

Example 2.2 The Bessel operator $B_{\alpha}: L^{2}\left(\mathbb{R}^{2}\right) \rightarrow L^{2}\left(\mathbb{R}^{2}\right)$ defined by $B_{\alpha} f=b_{\alpha} * f$ with $\hat{b}_{\alpha}(\xi)=\left(1+|\xi|^{2}\right)^{-\frac{\alpha}{2}}$ and $\alpha>0$ satisfies

$$
\left(B_{\alpha}^{*} B_{\alpha} f\right)^{\wedge}(\xi)=\left(1+|\xi|^{2}\right)^{-\alpha} \hat{f}(\xi)
$$

Proof It is known that $b_{\alpha}(x) \in L^{1}\left(\mathbb{R}^{2}\right)$ for $\alpha>0$ [11]. Hence, $\left(B_{\alpha} f\right)^{\wedge}(\xi)=\hat{b}_{\alpha}(\xi) \hat{f}(\xi)=(1+$ $\left.|\xi|^{2}\right)^{-\frac{\alpha}{2}} \hat{f}(\xi)$. For $f, g \in L^{2}\left(\mathbb{R}^{2}\right),\left\langle\left(B_{\alpha}^{*} B_{\alpha} f\right)^{\wedge}, \hat{g}\right\rangle=\left\langle B_{\alpha}^{*} B_{\alpha} f, g\right\rangle=\left\langle B_{\alpha} f, B_{\alpha} g\right\rangle=\left\langle\left(B_{\alpha} f\right)^{\wedge},\left(B_{\alpha} g\right)^{\wedge}\right\rangle=$ $\left\langle\left(1+|\xi|^{2}\right)^{-\alpha} \hat{f}(\xi), \hat{g}(\xi)\right\rangle$. Thus,

$$
\left(B_{\alpha}^{*} B_{\alpha} f\right)^{\wedge}(\xi)=\left(1+|\xi|^{2}\right)^{-\alpha} \hat{f}(\xi)
$$

To introduce the Riesz fractional integration transform, we define

$$
D=\left\{f \in L^{2}\left(\mathbb{R}^{2}\right), f \text { has compact support }\right\} \subseteq L^{1}\left(\mathbb{R}^{2}\right) \cap L^{2}\left(\mathbb{R}^{2}\right) .
$$

Then $D \subseteq L^{s}\left(\mathbb{R}^{2}\right)(1 \leq s \leq 2)$. For $f \in D$ and $0<\alpha<1$, the Riesz fractional integration transform is defined by

$$
I_{\alpha}(f)(x):=C_{\alpha} \int_{\mathbb{R}^{2}} \frac{f(y)}{|x-y|^{2-\alpha}} d y \in L^{2}\left(\mathbb{R}^{2}\right),
$$

where $C_{\alpha}$ is the normalizing constant [11]. In order to show $\left(I_{\alpha}^{*} I_{\alpha} f\right)^{\wedge}(\xi)=|\xi|^{-2 \alpha} \hat{f}(\xi)$ for $f \in D$ and $0<\alpha<1 / 2$, we need a lemma ([11], Lemma 2.15). 
Lemma 2.1 Let $S\left(\mathbb{R}^{2}\right)$ be the Schwartz space and $\Psi=\left\{\psi \in S\left(\mathbb{R}^{2}\right), \frac{\partial^{\beta}}{\partial x^{\beta}} \psi(0)=0, \beta \in \mathbb{Z}^{+} \times\right.$ $\left.\mathbb{Z}^{+}\right\}$with $\mathbb{Z}^{+}$being the non-negative integer set. Define $\Phi:=\{\varphi=\hat{\psi}, \psi \in \Psi\}$. Then with $\alpha>0$,

$$
\left(I_{\alpha} f\right)^{\wedge}(\xi)=|\xi|^{-\alpha} \hat{f}(\xi)
$$

holds for each $f \in \Phi$.

Example 2.3 The transform $I_{\alpha}$ defined by (2.1) satisfies $\left(I_{\alpha}^{*} I_{\alpha} f\right)^{\wedge}(\xi)=|\xi|^{-2 \alpha} \hat{f}(\xi)$ for $f \in D$ and $0<\alpha<\frac{1}{2}$.

Proof As proved in Examples 2.1, 2.2, it is sufficient to show that for $f \in D$,

$$
\left(I_{\alpha} f\right)^{\wedge}(\xi)=|\xi|^{-\alpha} \hat{f}(\xi)
$$

One proves (2.2) firstly for $f \in C_{0}^{\infty}\left(\mathbb{R}^{2}\right)$. Take $\mu(r) \in C^{\infty}([0, \infty))$ with $0 \leq \mu(r) \leq 1$ and

$$
\mu(r)= \begin{cases}1, & r \geq 2 \\ 0, & 0 \leq r \leq 1 .\end{cases}
$$

Define $\psi_{N}(\xi):=\mu(N|\xi|) \hat{f}(\xi)$. Then $\psi_{N}(\xi) \in \Psi$ and $f_{N}(x):=\check{\psi}_{N}(x)=\hat{\psi}_{N}(-x) \in \Phi$. By Lemma 2.1,

$$
\left(I_{\alpha} f_{N}\right)^{\wedge}(\xi)=|\xi|^{-\alpha} \hat{f}_{N}(\xi)
$$

Let $k(x)$ be the inverse Fourier transform of the function $1-\mu(|x|)$ and $k_{N}(x):=\frac{1}{N^{2}} k\left(\frac{x}{N}\right)$. Then $\int_{\mathbb{R}^{2}} k(x) d x=1$ and $f_{N}(x)=f(x)-k_{N} * f(x)$. Moreover, the classical approximation theorem [11] tells

$$
\lim _{N \rightarrow \infty}\left\|f_{N}-f\right\|_{p}=0
$$

for $p>1$. On the other hand, $\left\|I_{\alpha} f_{N}-I_{\alpha} f\right\|_{2}=\left\|I_{\alpha}\left(f_{N}-f\right)\right\|_{2} \leq C\left\|f_{N}-f\right\|_{\frac{2}{1+\alpha}}$ due to Theorem 16 [12, p.69]. Hence, $\lim _{N \rightarrow \infty}\left\|\left(I_{\alpha} f_{N}\right)^{\wedge}(\xi)-\left(I_{\alpha} f\right)^{\wedge}(\xi)\right\|_{2}=\lim _{N \rightarrow \infty}\left\|I_{\alpha} f_{N}-I_{\alpha} f\right\|_{2}=0$. That is,

$$
\lim _{N \rightarrow \infty}\left(I_{\alpha} f_{N}\right)^{\wedge}(\xi)=\left(I_{\alpha} f\right)^{\wedge}(\xi)
$$

in $L^{2}\left(\mathbb{R}^{2}\right)$ sense. Note that $\left\||\xi|^{-\alpha} \hat{f}_{N}(\xi)-|\xi|^{-\alpha} \hat{f}(\xi)\right\|_{2}^{2}=\int_{\mathbb{R}^{2}}|\xi|^{-2 \alpha}|\hat{f}(\xi)|^{2}[1-\mu(N|\xi|)]^{2} d \xi$; $|\xi|^{-2 \alpha}|\hat{f}(\xi)|^{2} \in L^{1}\left(\mathbb{R}^{2}\right)$ with $0<\alpha<\frac{1}{2}$ and $\lim _{N \rightarrow \infty}[1-\mu(N|\xi|)]=0$. Then

$$
\lim _{N \rightarrow \infty}\left\||\xi|^{-\alpha} \hat{f}_{N}(\xi)-|\xi|^{-\alpha} \hat{f}(\xi)\right\|_{2}=0
$$

thanks to the Lebesgue dominated convergence theorem, which means $\lim _{N \rightarrow \infty}|\xi|^{-\alpha} \times$ $\hat{f}_{N}(\xi)=|\xi|^{-\alpha} \hat{f}(\xi)$ in $L^{2}\left(\mathbb{R}^{2}\right)$ sense. This with (2.3), (2.4) shows (2.2) for $f \in C_{0}^{\infty}\left(\mathbb{R}^{2}\right)$.

In order to show (2.2) for $f \in D$, one can find $g \in C_{0}^{\infty}\left(\mathbb{R}^{2}\right)$ such that $\int_{\mathbb{R}^{2}} g(x) d x=1$ and $\lim _{N \rightarrow \infty}\left\|f * g_{N}-f\right\|_{p}=0(p \geq 1)$ by Theorem 4.2.1 in [13], where $g_{N}(\cdot)=N^{2} g(N \cdot)$. Since 
$f * g_{N} \in C_{0}^{\infty}\left(\mathbb{R}^{2}\right)$, the above proved fact says

$$
\left(I_{\alpha}\left(f * g_{N}\right)\right)^{\wedge}(\xi)=|\xi|^{-\alpha}\left(f * g_{N}\right)^{\wedge}(\xi) .
$$

The same arguments as (2.4) and (2.5) show that $\lim _{N \rightarrow \infty}\left(I_{\alpha}\left(f * g_{N}\right)\right)^{\wedge}(\xi)=\left(I_{\alpha} f\right)^{\wedge}(\xi)$ and $\lim _{N \rightarrow \infty}|\xi|^{-\alpha}\left(f * g_{N}\right)^{\wedge}(\xi)=|\xi|^{-\alpha} \hat{f}(\xi)$. Hence,

$$
\left(I_{\alpha} f\right)^{\wedge}(\xi)=|\xi|^{-\alpha} \hat{f}(\xi)
$$

This completes the proof of (2.2) for $f \in D$.

Next, we prove a lemma which will be used in the next section. For convenience, here and in what follows, we define $\mathcal{M}=N \cup M$ with $N=\mathbb{Z}^{2}, M:=\left\{\mu=(j, l, k, d): j \geq j_{0},-2^{j} \leq\right.$ $\left.l \leq 2^{j}-1, k \in \mathbb{Z}^{2}, d=0,1\right\}$. Then the shearlet system (introduced in Section 1) can be represented as $\left\{s_{\mu}: \mu \in \mathcal{M}\right\}$, where $s_{\mu}=\psi_{\mu}=\psi_{j, l, k}^{(d)}$ if $\mu \in M$, and $s_{\mu}=\varphi_{\mu}=\varphi_{j 0, k}$ if $\mu \in N$.

Lemma 2.2 Let $K$ satisfy $\left(K^{*} K f\right)^{\wedge}(\xi)=\left(b+|\xi|^{2}\right)^{-\alpha} \hat{f}(\xi)$ and $\left\{s_{\mu}, \mu \in \mathcal{M}\right\}$ be shearlets introduced in the first section. Define $\hat{\sigma}_{\mu}(\xi)=\left(b+|\xi|^{2}\right)^{\alpha} \hat{s}_{\mu}(\xi)$ and $U_{\mu}:=2^{-2 \alpha j} K \sigma_{\mu}$. Then $\left\|U_{\mu}\right\| \leq C$ and for $\mu \in \mathcal{M}$,

$$
\left\langle f, s_{\mu}\right\rangle=2^{2 \alpha j}\left\langle K f, U_{\mu}\right\rangle
$$

Proof By the Plancherel formula and the assumption $\hat{\sigma}_{\mu}(\xi)=\left(b+|\xi|^{2}\right)^{\alpha} \hat{s}_{\mu}(\xi)$, one knows that $\left\langle f, s_{\mu}\right\rangle=\left\langle\hat{f}, \hat{s}_{\mu}\right\rangle=\left\langle\hat{f}(\xi),\left(b+|\xi|^{2}\right)^{-\alpha} \hat{\sigma}_{\mu}(\xi)\right\rangle$. Moreover,

$$
\left\langle f, s_{\mu}\right\rangle=\left\langle\hat{f}(\xi),\left(K^{* \prime} K \sigma_{\mu}\right)^{\wedge}(\xi)\right\rangle=\left\langle f, K^{*} K \sigma_{\mu}\right\rangle=\left\langle K f, K \sigma_{\mu}\right\rangle=2^{2 \alpha j}\left\langle K f, U_{\mu}\right\rangle
$$

due to $\left(K^{*} K f\right)^{\wedge}(\xi)=\left(b+|\xi|^{2}\right)^{-\alpha} \hat{f}(\xi)$ and $U_{\mu}:=2^{-2 \alpha j} K \sigma_{\mu}$.

Next, one shows $\left\|U_{\mu}\right\| \leq C$. Note that $\left\|K \sigma_{\mu}\right\|^{2}=\left\langle K \sigma_{\mu}, K \sigma_{\mu}\right\rangle=\left\langle K^{*} K \sigma_{\mu}, \sigma_{\mu}\right\rangle=$ $\left\langle\left(K^{*} K \sigma_{\mu}\right)^{\wedge}, \hat{\sigma}_{\mu}\right\rangle,\left(K^{*} K \sigma_{\mu}\right)^{\wedge}=\left(b+|\xi|^{2}\right)^{-\alpha} \hat{\sigma}_{\mu}(\xi)$ and $\hat{\sigma}_{\mu}(\xi)=\left(b+|\xi|^{2}\right)^{\alpha} \hat{s}_{\mu}(\xi)$. Then $\left\|K \sigma_{\mu}\right\|^{2}=$ $\left\langle\hat{s}_{\mu}(\xi),\left(b+|\xi|^{2}\right)^{\alpha} \hat{s}_{\mu}(\xi)\right\rangle$ and

$$
\left\|U_{\mu}\right\|^{2}=2^{-4 \alpha j}\left\|K \sigma_{\mu}\right\|^{2}=2^{-4 \alpha j} \int_{\mathbb{R}^{2}}\left(b+|\xi|^{2}\right)^{\alpha}\left|\hat{s}_{\mu}(\xi)\right|^{2} d \xi
$$

Because supp $\hat{s}_{\mu} \subseteq C_{j}:=\left[-2^{2 j-1}, 2^{2 j-1}\right]^{2} \backslash\left[-2^{2 j-4}, 2^{2 j-4}\right]^{2}$, one receives $\left\|U_{\mu}\right\|^{2}=2^{-4 \alpha j} \int_{C_{j}}(b+$ $\left.|\xi|^{2}\right)^{\alpha}\left|\hat{s}_{\mu}(\xi)\right|^{2} d \xi \leq C$. This completes the proof of Lemma 2.2.

At the end of this section, we introduce two theorems which are important for our discussions. As in [8], we use $\operatorname{STAR}^{2}(A)$ to denote all sets $B \subseteq[0,1]^{2}$ with $C^{2}$ boundary $\partial B$ given by

$$
\beta(\theta)=\left(\begin{array}{l}
\rho(\theta) \cos \theta \\
\rho(\theta) \sin \theta
\end{array}\right)
$$

in a polar coordinate system. Here, $\rho(\theta) \leq \rho_{0}<1$ and $\left|\rho^{\prime \prime}(\theta)\right| \leq A$. Define $\varepsilon^{2}(A):=\{f=$ $\left.f_{0}+f_{1} X_{B}, B \in S T A R^{2}(A)\right\}$, where $f_{0}, f_{1} \in C_{0}^{2}\left([0,1]^{2}\right)$ are compactly supported on $[0,1]^{2}$. Let 


$$
\begin{gathered}
c_{\mu}:=\left\langle f, s_{\mu}\right\rangle, M_{j}:=\left\{(j, l, k, d),|k| \leq 2^{2 j+1},-2^{j} \leq l \leq 2^{j}-1, d=0,1\right\} \text { and } \\
R(j, \varepsilon)=\left\{\mu \in M_{j}:\left|c_{\mu}\right|>\varepsilon\right\} .
\end{gathered}
$$

Then with $\sharp R(j, \varepsilon)$ standing for the cardinality of $R(j, \varepsilon)$, the following conclusion holds [8].

Theorem 2.3 For $f \in \varepsilon^{2}(A), \sharp R(j, \varepsilon) \leq C \varepsilon^{-\frac{2}{3}}$ and

$$
\sum_{\mu \in M_{j}}\left|c_{\mu}\right|^{2} \leq C 2^{-2 j}
$$

Theorem 2.4 [14] Let $X \sim N(u, 1)$ and $t=\sqrt{2 \log \left(\eta^{-1}\right)}$ with $0<\eta \leq \frac{1}{2}$. Then

$$
E\left|T_{s}(X, t)-u\right|^{2}=\left[2 \log \left(\eta^{-1}\right)+1\right]\left(\eta+\min \left\{u^{2}, 1\right\}\right),
$$

where $N(u, 1)$ denotes the normal distribution with mean $u$ and variance 1 , while $T_{s}(y, t):=$ $\operatorname{sgn}(y)(|y|-t)_{+}$is the soft thresholding function.

\section{Main theorem}

In this section, we give an approximation result, which extends the result [8, Theorem 4.2] from the Radon transform to a family of linear operators. To do that, we introduce a set $\mathcal{N}(\varepsilon)$ of significant shearlet coefficients as follows. Let

$$
s_{1}=\frac{1}{\frac{9}{2}+6 \alpha} \log _{2}\left(\varepsilon^{-1}\right), \quad s_{2}=\frac{1}{\frac{3}{2}+2 \alpha} \log _{2}\left(\varepsilon^{-1}\right),
$$

and $j_{0}=\left\lceil s_{1}\right\rceil, j_{1}=\left\lceil s_{2}\right\rceil$. Define $\mathcal{N}(\varepsilon):=M(\varepsilon) \cup N(\varepsilon) \subseteq \mathcal{M}$, where

$$
\begin{aligned}
& N(\varepsilon)=\left\{\mu=k \in \mathbb{Z}^{2}:|k| \leq 2^{2 j_{0}+1}\right\} ; \\
& M(\varepsilon)=\left\{\mu=(j, l, k, d): j_{0} \leq j \leq j_{1},-2^{j} \leq l \leq 2^{j}-1,|k| \leq 2^{2 j+1}, d=0,1\right\} .
\end{aligned}
$$

Consider the model $Y=K f+\varepsilon W$ with $\left(K^{*} K f\right)^{\wedge}(\xi)=\left(b+|\xi|^{2}\right)^{-\alpha} \hat{f}(\xi)$. Lemma 2.2 tells that $y_{\mu}:=2^{2 \alpha j}\left\langle Y, U_{\mu}\right\rangle=\left\langle f, s_{\mu}\right\rangle+\varepsilon 2^{2 \alpha j} n_{\mu}$, where $n_{\mu}$ is Gaussian noise with zero mean and bounded variance $\sigma_{\mu}^{2}=\left\|U_{\mu}\right\|^{2} \leq C$ [15]. Let $c_{\mu}=\left\langle f, s_{\mu}\right\rangle$ and $\tilde{f}=\sum_{\mu \in \mathcal{N}(\varepsilon)} \tilde{c}_{\mu} s_{\mu}$ with

$$
\tilde{c}_{\mu}= \begin{cases}T_{s}\left(y_{\mu}, \varepsilon \sqrt{2 \log (\sharp \mathcal{N}(\varepsilon))} 2^{2 \alpha j} \sigma_{\mu}\right), & \mu \in \mathcal{N}(\varepsilon) ; \\ 0, & \text { otherwise, }\end{cases}
$$

where $T_{s}(y, t)$ is the soft thresholding function. Then the following result holds.

Theorem 3.1 Let $f \in \varepsilon^{2}(A)$ be the solution to $Y=K f+\varepsilon W$ with $\left(K^{*} K f\right)^{\wedge}(\xi)=(b+$ $\left.|\xi|^{2}\right)^{-\alpha} \hat{f}(\xi)$ and $\tilde{f}$ be defined as above. Then

$$
\sup _{f \in \varepsilon^{2}(A)} E\|\tilde{f}-f\|^{2} \leq C \log \left(\varepsilon^{-1}\right) \varepsilon^{\frac{2}{\frac{3}{2}+2 \alpha}} \quad(\varepsilon \rightarrow 0) .
$$


Proof Since $\left\{s_{\mu}, \mu \in \mathcal{M}\right\}$ is a Parseval frame, $f=\sum_{\mu \in \mathcal{M}} c_{\mu} s_{\mu}$ and $\tilde{f}=\sum_{\mu \in \mathcal{N}(\varepsilon)} \tilde{c}_{\mu} s_{\mu}, \tilde{f}-f=$ $\sum_{\mu \in \mathcal{M}}\left(\tilde{c}_{\mu}-c_{\mu}\right) s_{\mu}$. Moreover, $\|\tilde{f}-f\|^{2}=\sum_{\mu \in \mathcal{M}}\left|\tilde{c}_{\mu}-c_{\mu}\right|^{2}$ and

$$
E\|\tilde{f}-f\|^{2}=\sum_{\mu \in \mathcal{N}(\varepsilon)} E\left|\tilde{c_{\mu}}-c_{\mu}\right|^{2}+\sum_{\mu \in \mathcal{N}(\varepsilon)^{C}}\left|c_{\mu}\right|^{2} .
$$

In order to estimate $\sum_{\mu \in N(\varepsilon) C}\left|c_{\mu}\right|^{2}$, one observes $\sum_{\mu \in M_{j}}\left|c_{\mu}\right|^{2} \leq C 2^{-2 j}$ due to Theorem 2.3. Then $\sum_{j>j_{1}} \sum_{\mu \in M_{j}}\left|c_{\mu}\right|^{2} \leq C \sum_{j>j_{1}} 2^{-2 j} \leq C 2^{-2 j_{1}}$. By $2^{j_{1}} \lesssim \varepsilon^{-\frac{1}{2^{3}+2 \alpha}}$,

$$
\sum_{j>j_{1}} \sum_{\mu \in M_{j}}\left|c_{\mu}\right|^{2} \lesssim \varepsilon^{\frac{2}{\frac{3}{2}+2 \alpha}}
$$

(Here and in what follows, $A \lesssim B$ denotes $A \leq C B$ for some constant $C>0$ ).

Next, one considers $c_{\mu}$ for $j_{0} \leq j \leq j_{1}$ and $|k| \geq 2^{2 j+1}$. Note that $\left|\psi^{(d)}(x)\right| \leq C_{m}(1+|x|)^{-m}$ $(d=0,1, m=1,2, \ldots)$. Then $\left|\psi_{j, l, k}^{(d)}(x)\right| \leq C_{m} 2^{\frac{3}{2} j}\left(1+\left|B_{d}^{l} A_{d}^{j} x-k\right|\right)^{-m}$. Since $f \in \varepsilon^{2}(A)$, supp $f \subset$ $Q_{0}:=[0,1]^{2}$ and

$$
\mid\left\langle f, \psi_{j, l, k}^{(d)}|| \leq C_{m} 2^{\frac{3}{2} j}\|f\|_{\infty} \int_{Q_{0}}\left(1+\left|B_{d}^{l} A_{d}^{j} x-k\right|\right)^{-m} d x .\right.
$$

On the other hand, $\left|B_{d}^{l} A_{d}^{j} x\right| \leq\left\|B_{d}^{l} A_{d}^{j}\right\||x| \leq 2^{2 j}|x| \leq \sqrt{2} 2^{2 j}$ for $x \in Q_{0}$. Hence, $\left(1+\mid B_{d}^{l} A_{d}^{j} x-\right.$ $k \mid)^{-m} \leq\left(1+|k|-\left|B_{d}^{l} A_{d}^{j} x\right|\right)^{-m} \leq\left(|k|-\sqrt{2} 2^{2 j}\right)^{-m}$ for $|k| \geq 2^{2 j+1}$. Moreover, $\sum_{|k| \geq 2^{2 j+1}}\left|c_{\mu}\right|^{2} \leq$ $2^{3 j} \sum_{|k| \geq 2^{2 j+1}}\left(|k|-\sqrt{2} 2^{2 j}\right)^{-2 m}=2^{3 j} \sum_{n=1}^{\infty} \sum_{2^{2 j+n} \leq|k| \leq 2^{2 j+n+1}} 2^{-4 m j}\left(2^{n}-\sqrt{2}\right)^{-2 m} \lesssim 2^{3 j} 2^{-4 m j} \times$ $\sum_{n=1}^{\infty} 2^{2(2 j+n+1)}\left(2^{n}-\sqrt{2}\right)^{-2 m} \lesssim 2^{3 j} 2^{-2 j(2 m-2)}$, since $m$ can be chosen big enough. Therefore,

$$
\sum_{j=j_{0}}^{j_{1}} \sum_{l=-2^{j}}^{2^{j}-1} \sum_{|k| \geq 2^{2 j+1}}\left|c_{\mu}\right|^{2} \leq C_{m} \sum_{j=j_{0}}^{\infty} 2^{8 j} 2^{-4 m j} \lesssim 2^{-8 j_{0}} \leq 2^{-6 j_{0}} \lesssim \varepsilon^{\frac{2}{\frac{3}{2}+2 \alpha}}
$$

due to the choice of $j_{0}$. The similar (even simpler) arguments show $\sum_{|k| \geq 2^{2} j_{0}+1}\left|\left\langle f, \varphi_{j_{0}, k}\right\rangle\right|^{2} \lesssim$ $\varepsilon^{\frac{2}{2}+2 \alpha}$ with $\varphi_{j_{0}, k}(x)=2^{j_{0}} \varphi\left(2^{j_{0}} x-k\right)$. This with (3.2) and (3.3) leads to

$$
\sum_{\mu \in \mathcal{N}(\varepsilon)^{C}}\left|c_{\mu}\right|^{2} \lesssim \varepsilon^{\frac{2}{\frac{3}{2}+2 \alpha}}
$$

Finally, one estimates $\sum_{\mu \in \mathcal{N}(\varepsilon)} E\left|\tilde{c_{\mu}}-c_{\mu}\right|^{2}$. By the definition of $y_{\mu}, \varepsilon^{-1} 2^{-2 \alpha j} \sigma_{\mu}^{-1} y_{\mu} \sim$ $N\left(\varepsilon^{-1} 2^{-2 \alpha j} \sigma_{\mu}^{-1} c_{\mu}, 1\right)$. Applying Theorem 2.4 with $\eta^{-1}=\sharp \mathcal{N}(\varepsilon)$, one obtains that

$$
\begin{aligned}
& E\left|T_{s}\left[\varepsilon^{-1} 2^{-2 \alpha j} \sigma_{\mu}^{-1} y_{\mu}, \sqrt{2 \log (\sharp \mathcal{N}(\varepsilon))}\right]-\varepsilon^{-1} 2^{-2 \alpha j} \sigma_{\mu}^{-1} c_{\mu}\right|^{2} \\
& \quad=[2 \log (\sharp \mathcal{N}(\varepsilon))+1]\left[\frac{1}{\sharp \mathcal{N}(\varepsilon)}+\min \left\{\varepsilon^{-2} 2^{-4 \alpha j} \sigma_{\mu}^{-2} c_{\mu}^{2}, 1\right\}\right] .
\end{aligned}
$$


Hence, $E\left|T_{s}\left[y_{\mu}, \varepsilon 2^{2 \alpha j} \sigma_{\mu} \sqrt{2 \log (\sharp \mathcal{N}(\varepsilon))}\right]-c_{\mu}\right|^{2} \lesssim[2 \log (\sharp \mathcal{N}(\varepsilon))+1]\left[\varepsilon^{2^{2}} \frac{2^{4 \alpha j} \sigma_{\mu}^{2}}{\sharp \mathcal{N}(\varepsilon)}+\min \left\{c_{\mu}^{2}\right.\right.$, $\left.\left.\varepsilon^{2} 2^{4 \alpha j} \sigma_{\mu}^{2}\right\}\right]$. By $\tilde{c}_{\mu}=T_{s}\left[y_{\mu}, \varepsilon 2^{2 \alpha j} \sigma_{\mu} \sqrt{2 \log (\sharp \mathcal{N}(\varepsilon))}\right]$ for $\mu \in \mathcal{N}(\varepsilon)$, one knows that

$$
\begin{aligned}
E & \sum_{\mu \in \mathcal{N}(\varepsilon)}\left|\tilde{c_{\mu}}-c_{\mu}\right|^{2} \lesssim[2 \log (\sharp \mathcal{N}(\varepsilon))+1] \\
& \times\left[\varepsilon^{2} \sum_{\mu \in \mathcal{N}(\varepsilon)} \frac{2^{4 \alpha j} \sigma_{\mu}^{2}}{\sharp \mathcal{N}(\varepsilon)}+\sum_{\mu \in \mathcal{N}(\varepsilon)} \min \left\{c_{\mu}^{2}, \varepsilon^{2} 2^{4 \alpha j} \sigma_{\mu}^{2}\right\}\right] .
\end{aligned}
$$

Note that $\mathcal{N}(\varepsilon) \cap M_{j} \subset\left\{(j, l, k, d):|k| \leq 2^{2 j+1},|l| \leq 2^{j}\right\}$. Then $\sharp \mathcal{N}(\varepsilon) \leq C \sum_{j \leq j 1} 2^{5 j} \lesssim$ $2^{5 j_{1}} \lesssim \varepsilon^{-\frac{5}{2}+2 \alpha}$, and $\log (\sharp \mathcal{N}(\varepsilon)) \lesssim \frac{10}{\frac{3}{2}+2 \alpha} \log \left(\varepsilon^{-1}\right) \lesssim \log \left(\varepsilon^{-1}\right)$. Since $\left\{\sigma_{\mu}: \mu \in \mathcal{M}\right\}$ is uniformly bounded, $[2 \log (\sharp \mathcal{N}(\varepsilon))+1] \varepsilon^{2} \sum_{\mu \in \mathcal{N}(\varepsilon)} \frac{2^{4 \alpha j} \sigma_{\mu}^{2}}{\sharp \mathcal{N}(\varepsilon)} \lesssim \log \left(\varepsilon^{-1}\right) \varepsilon^{2} 2^{4 \alpha j_{1}}$. This with the choice of $2^{j_{1}}$ shows that

$$
[2 \log (\sharp \mathcal{N}(\varepsilon))+1] \varepsilon^{2} \sum_{\mu \in \mathcal{N}(\varepsilon)} \frac{2^{4 \alpha j} \sigma_{\mu}^{2}}{\sharp \mathcal{N}(\varepsilon)} \lesssim \log \left(\varepsilon^{-1}\right) \varepsilon^{\frac{3}{\frac{3}{2}+2 \alpha}} \lesssim \log \left(\varepsilon^{-1}\right) \varepsilon^{\frac{2}{\frac{3}{2}+2 \alpha}} .
$$

It remains to estimate $[2 \log (\sharp \mathcal{N}(\varepsilon))+1] \sum_{\mu \in \mathcal{N}(\varepsilon)} \min \left\{c_{\mu}^{2}, \varepsilon^{2} 2^{4 \alpha j} \sigma_{\mu}^{2}\right\}$. Clearly,

$$
\sum_{\mu \in \mathcal{N}(\varepsilon)} \min \left\{c_{\mu}^{2}, \varepsilon^{2} 2^{4 \alpha j}\right\}=\sum_{\left\{\mu \in \mathcal{N}(\varepsilon):\left|c_{\mu}\right| \geq 2^{2 \alpha j} \varepsilon\right\}} 2^{4 \alpha j} \varepsilon^{2}+\sum_{\left\{\mu \in \mathcal{N}(\varepsilon):\left|c_{\mu}\right|<2^{2 \alpha j} \varepsilon\right\}}\left|c_{\mu}\right|^{2} .
$$

By Theorem 2.3, $\sum_{\left\{\mu \in M_{j}:\left|c_{\mu}\right| \geq 2^{2 \alpha j} \varepsilon\right\}} 2^{4 \alpha j} \varepsilon^{2} \lesssim\left(2^{2 \alpha j} \varepsilon\right)^{-\frac{2}{3}} 2^{4 \alpha j} \varepsilon^{2} \lesssim 2^{\frac{8}{3} \alpha j} \varepsilon^{\frac{4}{3}}$. Hence,

$$
\sum_{\left\{\mu \in \mathcal{N}(\varepsilon):\left|c_{\mu}\right| \geq 2^{2 \alpha j} \varepsilon\right\}} 2^{4 \alpha j} \varepsilon^{2}=\sum_{j=j_{0}}^{j_{1}} \sum_{\left\{\mu \in M_{j:}:\left|c_{\mu}\right| \geq 2^{2 \alpha j} \varepsilon\right\}} 2^{4 \alpha j} \varepsilon^{2} \lesssim 2^{\frac{8}{3} \alpha j_{1}} \varepsilon^{\frac{4}{3}} .
$$

On the other hand, $\sum_{\left\{\mu \in \mathcal{N}(\varepsilon):\left|c_{\mu}\right|<2^{2 \alpha j} \varepsilon\right\}}\left|c_{\mu}\right|^{2}=\sum_{j=j_{0}}^{j_{1}} \sum_{n=0}^{\infty} \sum_{\left\{2^{2 \alpha j-n-1} \varepsilon<\left|c_{\mu}\right| \leq 2^{2 \alpha j-n} \varepsilon\right\}}\left|c_{\mu}\right|^{2}$. According to Theorem $2.3, \sharp R\left(j, 2^{2 \alpha j-n-1} \varepsilon\right) \lesssim 2^{-\frac{2}{3}(2 \alpha j-n-1)} \varepsilon^{-\frac{2}{3}}$ and

$$
\sum_{\left\{2^{2 \alpha j-n-1} \varepsilon<\left|c_{\mu}\right| \leq 2^{2 \alpha j-n} \varepsilon\right\}}\left|c_{\mu}\right|^{2} \lesssim 2^{-\frac{2}{3}(2 \alpha j-n-1)} \varepsilon^{-\frac{2}{3}} 2^{2(2 \alpha j-n)} \varepsilon^{2} \lesssim 2^{\frac{8}{3} \alpha j} 2^{-\frac{4}{3} n} 2^{\frac{2}{3}} \varepsilon^{\frac{4}{3}}
$$

Therefore,

$$
\sum_{\left\{\mu \in \mathcal{N}(\varepsilon):\left|c_{\mu}\right|<2^{2 \alpha j} \varepsilon\right\}}\left|c_{\mu}\right|^{2} \leq \sum_{j=j_{0}}^{j_{1}} \sum_{n=0}^{\infty} 2^{\frac{8}{3} \alpha j^{-\frac{4}{3} n}} \varepsilon^{\frac{4}{3}} \leq 2^{\frac{8}{3} \alpha j_{1}} \varepsilon^{\frac{4}{3}} .
$$

Combining this with (3.7) and (3.8), one knows that $\sum_{\mu \in \mathcal{N}(\varepsilon)} \min \left\{c_{\mu}^{2}, \varepsilon^{2} 2^{4 \alpha j}\right\} \lesssim 2^{\frac{8}{3} \alpha j_{1}} \varepsilon^{\frac{4}{3}}$. Furthermore,

$$
\sum_{\mu \in \mathcal{N}(\varepsilon)} \min \left\{c_{\mu}^{2}, \varepsilon^{2} 2^{4 \alpha j}\right\} \lesssim \varepsilon^{\frac{2}{2 \alpha+\frac{3}{2}}}
$$


thanks to $2^{j_{1}} \lesssim \varepsilon^{-\frac{1}{2 \alpha+\frac{3}{2}}}$. Now, it follows from (3.5), (3.6) and (3.9) that

$$
\sum_{\mu \in \mathcal{N}(\varepsilon)} E\left|\tilde{c_{\mu}}-c_{\mu}\right|^{2} \lesssim \log \left(\varepsilon^{-1}\right) \varepsilon^{\frac{2}{2 \alpha+\frac{3}{2}}}
$$

This with (3.1) and (3.4) leads to the desired conclusion $\sup _{f \in \varepsilon^{2}(A)} E\|\tilde{f}-f\|^{2} \leq C \log \left(\varepsilon^{-1}\right) \times$ $\varepsilon^{\frac{2}{\frac{3}{2}+2 \alpha}}$. The proof is completed.

\section{Competing interests}

The authors declare that they have no competing interests.

\section{Authors' contributions}

LH and YL finished this work together. Two authors read and approved the final manuscript.

\section{Author details}

'Department of Applied Mathematics, Beijing University of Technology, Beijing, 100124, P.R. China. ${ }^{2}$ Department of Basic Courses, Beijing Union University, Beijing, 100101, P.R. China.

\section{Acknowledgements}

This work is supported by the National Natural Science Foundation of China (No. 11271038) and the Natural Science Foundation of Beijing (No. 1082003).

Received: 14 August 2012 Accepted: 18 December 2012 Published: 7 January 2013

\section{References}

1. Natterer, F: The Mathematics of Computerized Tomography. Wiley, New York (1986)

2. Natterer, F, Wübbeling, F: Mathematical Methods in Image Reconstruction. SIAM Monographs on Mathematical Modeling and Computation. SIAM, Philadelphia (2001)

3. Candés, EJ, Donoho, DL: Continuous curvelet transform: II. Discretization and frames. Appl. Comput. Harmon. Anal. 19, 198-222 (2005)

4. Candés, EJ, Donoho, DL: New tight frames of curvelets and optimal representations of objects with $C^{2}$ singularities. Commun. Pure Appl. Math. 57, 219-266 (2004)

5. Candés, EJ, Donoho, DL: Recovering edges in ill-posed inverse problems: optimality of curvelet frames. Ann. Stat. 30, 784-842 (2002)

6. Easley, G, Labate, D, Lim, WQ: Sparse directional image representations using the discrete shearlet transform. Appl. Comput. Harmon. Anal. 25, 25-46 (2008)

7. Guo, K, Labate, D: Optimally sparse multidimensional representation using shearlets. SIAM J. Math. Anal. 39, 298-318 (2007)

8. Colonna, F, Easley, G, Guo, K, Labate, D: Radon transform inversion using the shearlet representation. Appl. Comput. Harmon. Anal. 29, 232-250 (2010)

9. Lee, NY, Lucier, BJ: Wavelet methods for inverting the Radon transform with noisy data. IEEE Trans. Image Process. 10, 1079-1094 (2001)

10. Strichartz, RS: Radon inversion-variations on a theme. Am. Math. Mon. 89, 377-384 (1982)

11. Samko, SG: Hypersingular Integrals and Their Applications. Taylor \& Francis, London (2002)

12. Zhou, MQ: Harmonic Analysis. Beijing Normal University Press, Beijing (1999)

13. Lu, SZ, Wang, KY: Real Analysis. Beijing Normal University Press, Beijing (2005)

14. Donoho, DL, Johnstone, IM: Ideal spatial adaptation via wavelet shrinkage. Biometrika 81, 425-455 (1994)

15. Johnstone, IM: Gaussian Estimation: Sequence and Wavelet Models. http://www-stat.stanford.edu/imj/GE12-27-11 (1999)

doi:10.1186/1029-242X-2013-11

Cite this article as: Hu and Liu: Shearlet approximations to the inverse of a family of linear operators. Journal of Inequalities and Applications 2013 2013:11. 